\title{
Dichroism optical-resolution photoacoustic microscopy
}

S. Hu, K. Maslov, Ping Yan, Jin-Moo Lee, L. V. Wang

S. Hu, K. Maslov, Ping Yan, Jin-Moo Lee, L. V. Wang, "Dichroism opticalresolution photoacoustic microscopy," Proc. SPIE 8223, Photons Plus Ultrasound: Imaging and Sensing 2012, 82233T (23 February 2012); doi: 10.1117/12.909401

SPIE. Event: SPIE BiOS, 2012, San Francisco, California, United States 


\title{
Dichroism Optical-resolution Photoacoustic Microscopy
}

\author{
S. Hu ${ }^{1, \uparrow}$, K. Maslov ${ }^{1, \uparrow}$, Ping Yan ${ }^{2}$, Jin-Moo Lee ${ }^{2}$, and L. V. Wang ${ }^{1, *}$ \\ ${ }^{1}$ Department of Biomedical Engineering, Washington University in St. Louis, St. Louis, MO 63130, USA \\ ${ }^{2}$ Department of Neurology, Washington University School of Medicine, St. Louis, MO, USA \\ ${ }^{\dagger}$ Authors contributed equally \\ *Correspondence should be addressed to LVW (1hwang@biomed.wustl.edu).
}

\begin{abstract}
We have developed dichroism optical-resolution photoacoustic microscopy, capable of imaging polarization-dependent optical absorption (i.e., dichroism) with excellent specificity. This technical innovation enriches molecular photoacoustic contrasts and holds particular potential for detecting amyloid-associated neurodegenerative and cardiovascular diseases.
\end{abstract}

Keywords: Optical-resolution photoacoustic microscopy, polarization-dependent optical absorption (dichroism), amyloid plaque, Alzheimer's disease.

\section{INTRODUCTION}

Dichroism, anisotropy in optical absorption, encodes rich information on molecular conformation and structural alignment. There are three strong motivations for targeting dichroism. First, dichroism is a unique molecular signature with an ideal specificity. Second, the distinct absorption responses of dichroic molecules to differently polarized optical irradiations enable differential detection, which can eliminate the non-dichroic background and greatly enhance sensitivity. The third and most important motivation is that amyloid, a hallmark of a wide spectrum of protein aggregation disorders, is dichroic. Photoacoustics, providing highly sensitive and accurate quantification of optical absorption [1-4], has decided advantages in detecting dichroism contrasts of biomolecules; however, to date there has been no such experimental demonstration. To this end, we have developed dichroism optical-resolution photoacoustic microscopy (DOR-PAM). Our preliminary results show its extraordinary sensitivity and specificity in probing the linear dichroism of Congo Red (CR)-labeled amyloid plaques ex vivo. An in vivo study is underway.

\section{METHODS AND MATERIALS}

In the DOR-PAM system (Fig. 1), the output intensity of a Q-switched laser (pulse width: $1.8 \mathrm{ns;}$ wavelength: $532 \mathrm{~nm}$; repetition rate: $0-50 \mathrm{kHz}$ ) is regulated by a neutral density filter (NDF) to meet the safety standards of the American National Standards Institute. Then, the attenuated linearly polarized laser beam passes through a half-wave plate (HWP) and bifurcates into two orthogonally polarized beams through a polarizing beam splitter (PBS). The splitting ratio can be precisely regulated by rotating the fast axis of the HWP with respect to the initial laser polarization direction, to balance the pulse energies of the two daughter beams. Two electro-optic modulators (EOM) are inserted into the two split optical paths, respectively, as two optical switches. Thus, the polarization of the recombined laser beam from the second PBS can be switched pulsewise between the two orthogonal states. A beam sampler (BS) and a photodiode (PD) are positioned before a single-mode fiber coupler (FC) to monitor the fluctuation in laser energy. To maintain the polarization of the recombined laser beam, a polarization-maintaining fiber (PMF) is utilized and its fast and slow axes are aligned with the two orthogonal states, respectively. To achieve optical resolution along the lateral dimensions, two identical microscope objectives are employed to map the fiber output spot into objects to be imaged through the center hole of a ring transducer. Two-dimensional raster scanning of the imaging head (the red dashed box in Fig. 1) combined with time-resolved acoustic detection provides volumetric imaging of tissue dichroism.

Photons Plus Ultrasound: Imaging and Sensing 2012, edited by Alexander A. Oraevsky, Lihong V. Wang, Proc. of SPIE Vol. 8223, 82233T - (C) 2012 SPIE · CCC code: 1605-7422/12/\$18 - doi: 10.1117/12.909401 


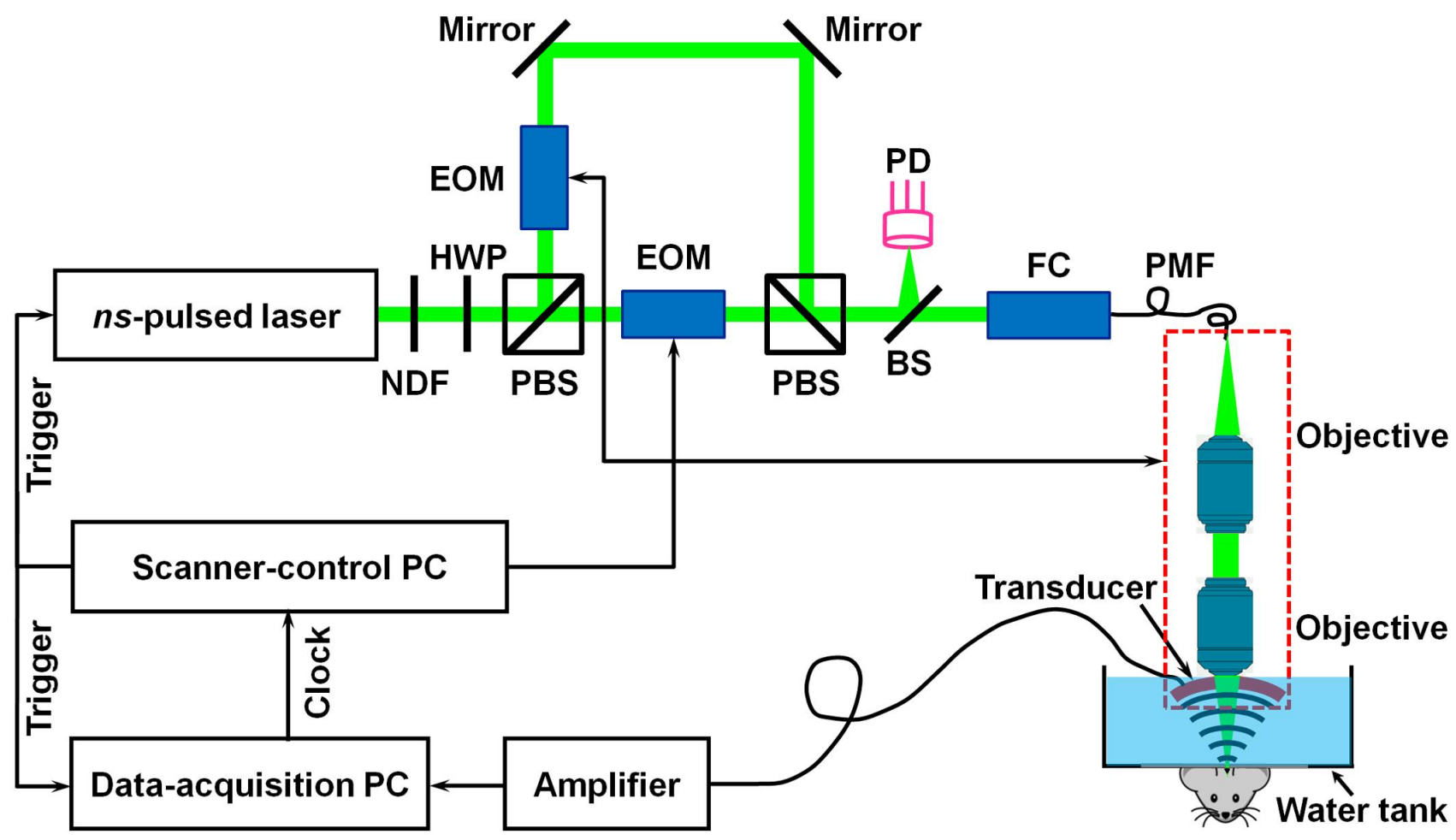

Fig. 1. Schematic of the DOR-PAM system.

\section{RESULTS}

Congo Red is a commonly used amyloid dye to stain fibrils in amyloid plaques, wherein the dye molecules intercalate between $\beta$-pleated sheets fibrils and exhibit strong linear dichroism [5]. This dichroism can be utilized as a specific molecular contrast to differentiate amyloid plaques from the non-dichroic background. As a proof-of-principle study, we stained a brain section from an APP/PS1 mouse (mouse model of Alzheimer's disease) with CR, which targets amyloid plaques. Under each of the two linearly polarized optical irradiations at $532 \mathrm{~nm}$, the CR-stained amyloid plaques showed similar optical absorption intensity (Figs. 2A-D). In contrast, subtraction of the two images acquired with the orthogonally polarized optical irradiations completely removed the non-dichroic background and highlighted the dichroic amyloid plaques (Fig. 2E, F).

Previously, we demonstrated amyloid plaque imaging through a cranial window, using conventional optical-resolution photoacoustic microscopy (OR-PAM) (Fig. 3) [6]. However, such a window may adversely influence the behavior of underlying brain tissues and poses a practical limit on the current gold standard of two-photon microscopy. DOR-PAM, with significantly enhanced sensitivity and specificity over conventional OR-PAM, is expected to enable visualizing individual amyloid plaques through intact skulls. This technical innovation will create a powerful in vivo screening tool for anti-amyloid drugs. 

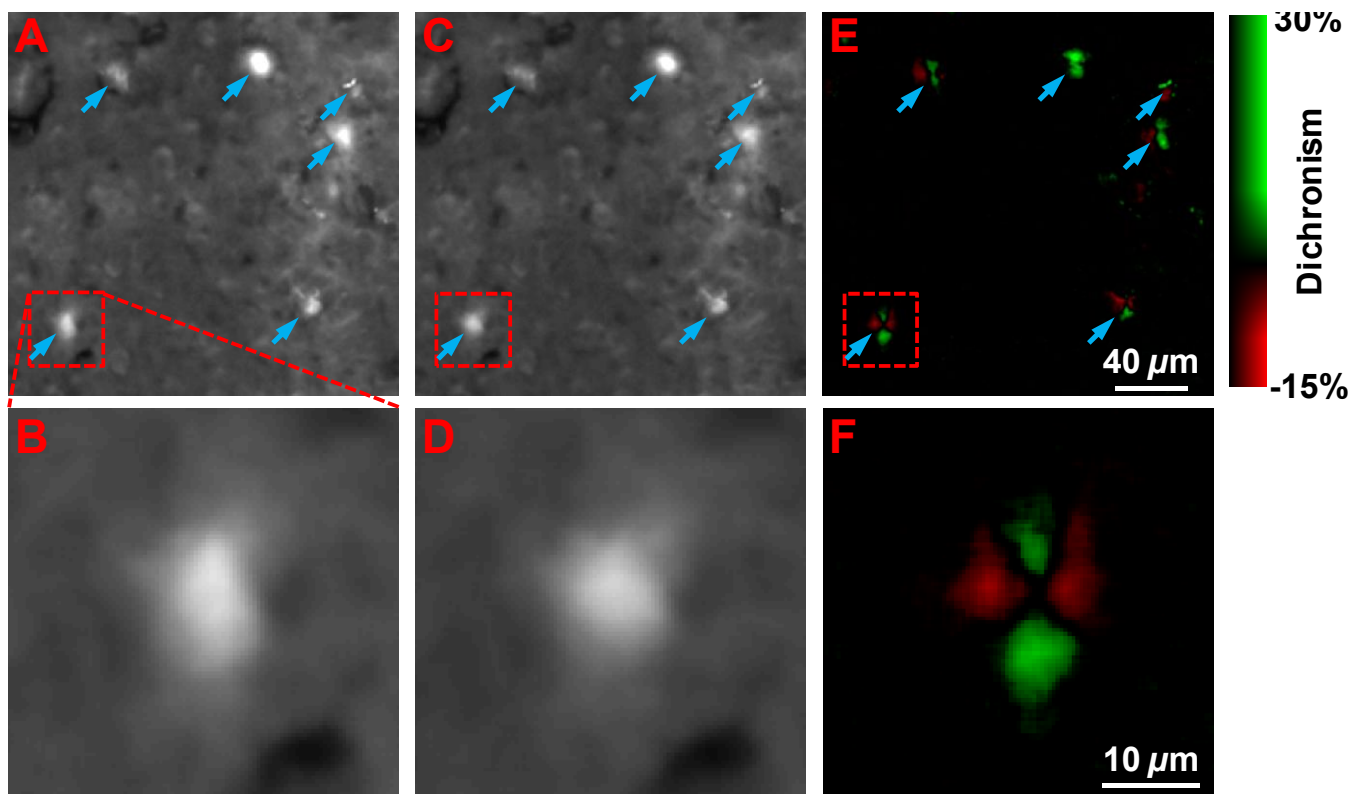

Fig. 2. DOR-PAM of amyloid plaques in an APP/PS1 mouse brain section stained with Congo Red (CR). (A, C) The DOR-PAM images acquired with each of the two orthogonally polarized optical irradiations, respectively. (B, D) The close-ups of the boxed area in Panels A and C, respectively. (E) The subtraction of (A) and (C), which eliminates the non-dichroic background and highlights the dichroic contrast of the amyloid plaques. (F) The close-up of the boxed area in Panels E, showing the bipolar dichroism pattern of the amyloid plaque. The blue arrows in Panels A, C and E indicate the CR-stained amyloid plaques.
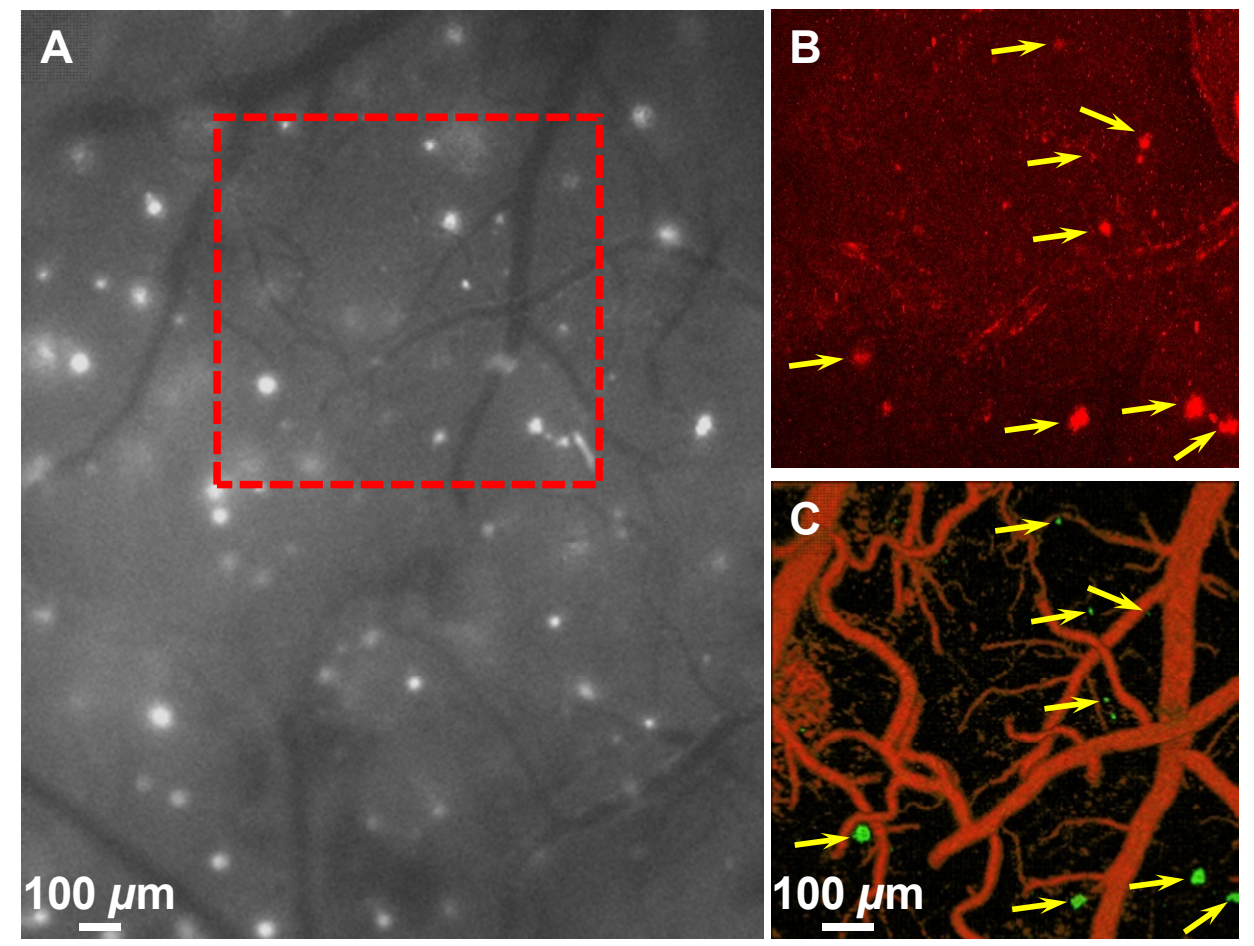

Fig. 3. In vivo conventional OR-PAM of a Congo-red stained APP/PS1 mouse cortex through a cranial window. (A) Exposed cortical brain region imaged using conventional fluorescence microscopy through the cranial window. The region of interest marked by a red dashed box was imaged by two-photon microscopy in (B), and dual-wavelength ORPAM in (C). In the dual-contrast OR-PAM image, amyloid plaques are colored green and blood vessels are colored red. 


\section{CONCLUSION}

We have developed DOR-PAM with orthogonally polarized optical irradiation to detect the molecular-specific linear dichroism contrast of amyloid plaques. This technical innovation adds a new molecular contrast to photoacoustic imaging, and holds great potential in studying the pathophysiology of a spectrum of amyloid-associated neurodegenerative and cardiovascular diseases.

\section{ACKNOWLEDGMENTS}

This work was sponsored by National Institutes of Health Grants R01 EB000712, R01 EB008085, R01 CA134539, U54 CA136398, R01 CA157277, R01 EB010049, and R01 CA159959. L.V.W. has financial interests in Microphotoacoustics, Inc. and Endra, Inc., which, however, did not support this work.

\section{REFERENCES}

1. S. Hu, K. Maslov, and L. V. Wang, "Second-generation optical-resolution photoacoustic microscopy with improved sensitivity and speed," Opt. Lett. 36, 1134-1136 (2011).

2. S. Hu, and L. V. Wang, "Photoacoustic imaging and characterization of the microvasculature," J. Biomed. Opt. 15, $011101(2010)$.

3. S. Hu, and L. V. Wang, "Neurovascular photoacoustic tomography," Front. Neuroenerg. 2, doi:10.3389/fnene.2010.00010 (2010).

4. L. V. Wang, "Multiscale photoacoustic microscopy and computed tomography," Nat. Photon. 3, 503-509 (2009).

5. L. W. Jin, K. A. Claborn, M. Kurimoto, M. A. Geday, I. Maezawa, F. Sohraby, M. Estrada, W. Kaminksy, and B. Kahr, "Imaging linear birefringence and dichroism in cerebral amyloid pathologies," Proc. Natl. Acad. Sci. USA 100, 15294-15298 (2003).

6. S. Hu, P. Yan, K. Maslov, J.-M. Lee and L. V. Wang, "Intravital imaging of amyloid plaques in a transgenic mouse model using optical-resolution photoacoustic microscopy," Opt. Lett. 34(24), 3899-3901 (2009). 